\title{
Study of the biodistribution of fluorescein in glioma-infiltrated mouse brain and histopathological correlation of intraoperative findings in high-grade gliomas resected under fluorescein fluorescence guidance
}

\author{
Roberto Jose Diaz, MD, PhD, ${ }^{1-3}$ Roberto Rey Dios, MD, PhD, ${ }^{5}$ Eyas M. Hattab, MBBS, ${ }^{6}$ \\ Kelly Burrell, MSc, ${ }^{1}$ Patricia Rakopoulos, MSc, ${ }^{1}$ Nesrin Sabha, ${ }^{1}$ Cynthia Hawkins, MD, PhD, ${ }^{1}$ \\ Gelareh Zadeh, MD, PhD, ${ }^{3,4}$ James T. Rutka, MD, PhD, ${ }^{1-3}$ and Aaron A. Cohen-Gadol, MD, MSc ${ }^{7}$
}

${ }^{1}$ The Hospital for Sick Children, Arthur and Sonia Labatt Brain Tumour Research Centre; 2Department of Laboratory Medicine \& Pathobiology and ${ }^{3}$ Division of Neurosurgery, Department of Surgery, University of Toronto; ${ }^{4}$ Toronto Western Hospital University Health Network, Toronto, Ontario, Canada; ${ }^{5}$ Department of Neurosurgery, University of Mississippi Medical Center, Jackson, Mississippi; ${ }^{6}$ Department of Pathology and Laboratory Medicine, Indiana University; and ${ }^{7}$ Goodman Campbell Brain and Spine, Department of Neurological Surgery, Indiana University, Indianapolis, Indiana

OBJECT Intravenous fluorescein sodium has been used during resection of high-grade gliomas to help the surgeon visualize tumor margins. Several studies have reported improved rates of gross-total resection (GTR) using high doses of fluorescein sodium under white light. The recent introduction of a fluorescein-specific camera that allows for high-quality intraoperative imaging and use of very low dose fluorescein has drawn new attention to this fluorophore. However, the ability of fluorescein to specifically stain glioma cells is not yet well understood.

METHODS The authors designed an in vitro model to assess fluorescein uptake in normal human astrocytes and U251 malignant glioma cells. An in vivo experiment was also subsequently designed to study fluorescein uptake by intracranial U87 malignant glioma xenografts in male nonobese diabetic/severe combined immunodeficient mice. A genetically induced mouse glioma model was used to adjust for the possible confounding effect of an inflammatory response in the xenograft model. To assess the intraoperative application of this technology, the authors prospectively enrolled 12 patients who underwent fluorescein-guided resection of their high-grade gliomas using low-dose intravenous fluorescein and a microscope-integrated fluorescence module. Intraoperative fluorescent and nonfluorescent specimens at the tumor margins were randomly analyzed for histopathological correlation.

RESULTS The in vitro and in vivo models suggest that fluorescein demarcation of glioma-invaded brain is the result of distribution of fluorescein into the extracellular space, most likely as a result of an abnormal blood-brain barrier. Glioblastoma tumor cell-specific uptake of fluorescein was not observed, and tumor cells appeared to mostly exclude fluorescein. For the 12 patients who underwent resection of their high-grade gliomas, the histopathological analysis of the resected specimens at the tumor margin confirmed the intraoperative fluorescent findings. Fluorescein fluorescence was highly specific (up to $90.9 \%$ ) while its sensitivity was $82.2 \%$. False negatives occurred due to lack of fluorescence in areas of diffuse, low-density cellular infiltration. Margins of contrast enhancement based on intraoperative MRI-guided StealthStation neuronavigation correlated well with fluorescent tumor margins. GTR of the contrast-enhancing area as guided by the fluorescent signal was achieved in $100 \%$ of cases based on postoperative MRI.

CONCLUSIONS Fluorescein sodium does not appear to selectively accumulate in astrocytoma cells but in extracellular tumor cell-rich locations, suggesting that fluorescein is a marker for areas of compromised blood-brain barrier within high-grade astrocytoma. Fluorescein fluorescence appears to correlate intraoperatively with the areas of MR enhancement, thus representing a practical tool to help the surgeon achieve GTR of the enhancing tumor regions.

http://thejns.org/doi/abs/10.3171/2015.2.JNS132507

KEY WORDS fluorescein; glioma cells; uptake; distribution; marker; resection; oncology

ABBREVIATIONS BBB = blood-brain barrier; GTR = gross-total resection; IQR = interquartile range; NHA = normal human astrocyte; PBS = phosphate-buffered saline; PDGF = platelet-derived growth factor; PDGFB = PDGF beta; PDGFR = PDGF receptor; PDGFRA = PDGFR alpha; RCAS = replication-competent avian sarcoma-leukosis; $\mathrm{RFP}=$ red fluorescent protein; tva = tumor virus $A$.

ACCOMPANYING EDITORIAL See pp 1356-1359. DOI: 10.3171/2014.10.JNS141788.

SUBMITTED November 12, 2013. ACCEPTED February 23, 2015.

INCLUDE WHEN CITING Published online April 3, 2015; DOI: 10.3171/2015.2.JNS132507.

DISCLOSURE Aaron A. Cohen-Gadol has a consulting agreement with Carl Zeiss Meditec AG. The compensation from this arrangement is donated to a not-for-profit educational organization, The Neurosurgical Atlas. 
I NTRAVENOUS administration of sodium fluorescein (fluorescein) during resection of malignant gliomas results in demarcation of the regions of brain with tumor infiltration, highlighted by enhanced fluorescein uptake and slower fluorescein clearance. ${ }^{16,19,24}$ Fluorescein (MW 376 D) is an organic fluorescent dye with peak excitation at $490 \mathrm{~nm}$ and emission between 500 and $550 \mathrm{~nm}$, dependent on $\mathrm{pH}$ and the ionic strength of the solution..$^{25}$ This dye can be safely administered intravenously in humans and has been used clinically in multiple applications..$^{20}$ The use of fluorescein as a tumor marker for resection has been widely investigated for multiple tumor types, including skull base tumors ${ }^{6}$ metastatic brain tumors, ${ }^{21}$ and high-grade gliomas. ${ }^{10,11,16,23,24}$ Fluorescein has not been previously demonstrated as an agent that bioaccumulates in a glioblastoma flank xenograft model. ${ }^{10}$ However, conjugation of fluorescein to albumin demarcates glioblastoma tumors in humans and animal models. ${ }^{9,12}$ Because the mechanism of tumor delineation by unconjugated fluorescein in the brain is not clear, we hypothesized that the enhanced uptake and slow washout of fluorescein is due to selective uptake of fluorescein into glioma cells within the brain parenchyma. Furthermore, to understand the clinical correlation of our laboratory findings, we explored the accuracy of intraoperative fluorescein fluorescence to predict the extent of tumor infiltration by high-grade glioma cells.

\section{Laboratory Methods}

\section{In Vitro Assessment of Fluorescein Uptake by Astrocytes and Glioma Cells}

To determine if fluorescein bioaccumulates in normal human astrocytes (NHAs) and U251 malignant glioma cells when it is present in the extracellular space, we incubated NHAs and U251 cells with fluorescein and assessed cellular uptake by microscopy. NHAs were cultured in astrocyte growth media (Lonza), and U251 cells were cultured in DMEM with $10 \%$ fetal bovine serum (Wisent Inc.). Cells were grown on coverslips at a density of 98 cells per $\mathrm{mm}^{2}$ for 24 hours under $5 \% \mathrm{CO}_{2}$ atmosphere at $37^{\circ} \mathrm{C}$. The medium was then replaced with medium containing $500 \mathrm{ng} / \mathrm{ml}$ sodium fluorescein (AK-Fluor, Akorn Inc.) and cells were incubated for $5,15,30$, or 60 minutes. The medium was then removed, and the cells were immediately fixed with $4 \%$ (vol/vol) paraformaldehyde solution (16\% paraformaldehyde solution [Electron Microscopy Sciences] diluted in phosphate-buffered saline [PBS]) at room temperature for 10 minutes. The coverslips were then mounted on a slide with hard-set mounting medium containing DAPI (Vector Laboratories Inc.). Cells were imaged using an Olympus $1 \times 81$ spinning disc confocal microscope (Olympus Canada Inc.) with a Yokogawa scan head (Yokogawa Corp. of America). For fluorescein visualization, a 491-nm excitation laser with a 525/50-nm bandpass filter was used. The experiment was repeated 3 times.

\section{In Vivo Imaging of Intracranial Xenograft With Intravenous Fluorescein Administration}

Intracranial U87 malignant glioma xenografts were established in nonobese diabetic/severe combined immu- nodeficient (NOD/SCID) male mice $(\mathrm{n}=4)$. An intracranial imaging window placed over the region of injection has been previously described. ${ }^{4}$ The U87 cells used in the xenografts were engineered to constituently express pCAG mCherry:IRES:Puro using Fugene HD at a ratio of 3:1. Seven days after intracranial xenograft implantation, the mice were anesthetized, and a tail vein injection of Alexa Fluor 647 10-kD dextran (Invitrogen) at a dose of $0.6 \mathrm{mg} / \mathrm{kg}$ was administered; a second intravenous injection of fluorescein was given immediately at a dose of $7.7 \mathrm{mg} / \mathrm{kg}$. The mice were then inverted onto the imaging platform of the 2-photon laser confocal microscope (LSM510 2-Photon Laser Confocal, Zeiss). Using 5x, 10×, and $20 \times$ long working distance air objectives, images of mCherry-positive tumor mass, Alexa Fluor 647 dextranpositive cerebral vasculature, and fluorescein distribution were obtained at $5,10,15,20$, and 25 minutes after fluorescein injection. The excitation lasers and filter sets used for imaging are summarized in Table 1 . Fluorescence intensity measurements across a region of interest in both the visible tumor area and adjacent brain parenchyma were processed using Zen lite software (Carl Zeiss AG), and comparison was made between the red (tumor cells), green (fluorescein), and blue (dextran) emission channels.

\section{In Vivo Imaging of RCAS/tva-Derived Gliomas With Intravenous Fluorescein Administration}

Given the possible confounding effect of an inflammatory response in the xenograft glioma model at the site of tumor implantation, we proceeded to test fluorescein biodistribution using the intracranial window method described above in a genetically induced mouse glioma model. Mouse GFAP-tva pups were derived from mating pairs obtained from the Jackson Laboratory. At postnatal Day 3, cells were injected to a depth of $3 \mathrm{~mm}$ in the right frontal lobe with $2 \mu$ of PBS $(n=1)$ or with approximately $2 \times 10^{5} \mathrm{DF} 1$ cells producing RCAS-PDGFBRFP virus $(n=11)$. Glial cells in these mice express the tva receptor under a GFAP promoter, leading to selective integration and expression of the packaged cassette containing the platelet-derived growth factor beta (PDGFB) gene and red fluorescent protein (RFP) gene in cells that express GFAP. The viral plasmid was obtained from Dr. Oren Becher (Duke University) and viral preparation was performed according to published protocols. ${ }^{3}$ Four to 6 weeks after viral injection, a cranial window was made over the right frontal lobe and the brain surface was imaged as described for xenografts above. Alexa Fluor 647 $10-\mathrm{kD}$ dextran (Invitrogen) at a dose of $0.6 \mathrm{mg} / \mathrm{kg}$ was administered, followed immediately by fluorescein at a dose of $7.7 \mathrm{mg} / \mathrm{kg}$ diluted in $0.9 \% \mathrm{NaCl}$ solution to achieve an administration volume of $0.2 \mathrm{ml}$. The fluorescent dextran allowed blood vessel visualization and internal control for assessment of blood-brain barrier (BBB) permeability. Images of the brain surface were obtained at $0-, 5-, 10-$, 15-, 20-, and 25-minute time points using the 2-photon laser confocal microscope with parameters as described in Table 1. Animals were killed with $\mathrm{CO}_{2}$, and their brains were excised and fixed in $3.7 \%$ (vol/ $/ \mathrm{vol})$ formaldehyde in PBS prior to embedding in paraffin.

Coronal sections of the frontal lobes were stained with 
TABLE 1. Excitation lasers and filter sets used to image fluorescein, mCherry-expressing U87 glioma cells or RFP-expressing glioma cells, and intravascular Alexa Fluor 647 dextran

\begin{tabular}{cccc}
\hline & \multicolumn{3}{c}{ Fluorochrome } \\
\cline { 2 - 4 } Variable & Fluorescein & mCherry/RFP & $\begin{array}{c}\text { Alexa Fluor 647 } \\
\text { Dextran }\end{array}$ \\
\hline Excitation laser & $488 \mathrm{~nm}$ & $543 \mathrm{~nm}$ & $633 \mathrm{~nm}$ \\
\hline Collection filter & $500-550 \mathrm{~nm}$ & $565-615 \mathrm{~nm}$ & $650-710 \mathrm{~nm}$ \\
\hline
\end{tabular}

$\mathrm{H} \& \mathrm{E}$ for confirmation of glioma formation. The presence of tumor cells at the imaging site was further confirmed by immunohistochemistry for GFAP (University Health Network Pathology Research Program). To test for the overexpression of platelet-derived growth factor (PDGF) by viral transduction, the expression of platelet-derived growth factor receptor alpha (PDGFRA), which is known to be upregulated in response to PDGFB, was assessed by immunohistochemical analysis. Heat antigen retrieval was performed in citrate buffer. Rabbit anti-PDGFRA antibody (Cell Signaling Technologies) was used at a 1:200 dilution in $10 \%$ goat serum solution with overnight incubation at room temperature. Detection was performed with avidin-biotin-horseradish peroxidase complex (ABC, Vector Laboratories) followed by diaminobenzidine as the chromogen. Nuclei were counterstained with hematoxylin. Immunolabeled sections were imaged on an Olympus $1 \times$ 73 microscope.

\section{Laboratory Results}

The uptake of fluorescein in cultured normal fetal human astrocytes and glioblastoma cells was investigated to determine if a cell type-specific bioaccumulation of fluorescein was present in glioblastoma cells. We demonstrated a slight time-dependent increase in fluorescein signal within NHAs (Fig. 1) and a relative exclusion of fluorescein signal in U251 cells, even after 60 minutes of exposure (Fig. 2). Since cellular uptake of fluorescein may be different in cells within a native environment, we proceeded to image the distribution of fluorescein in relation to the location of tumor cells in intracranial xenografts. Cranial window imaging revealed parenchymal uptake of fluorescein within regions of tumor infiltration (Fig. 3 upper). However, despite the parenchymal fluorescein signal observed in the region of the tumor, there was no specific uptake of fluorescein into U87-mCherry glioma cells in vivo (Fig. 3 lower). In regions of closely packed tumor cells there was a relative reduction in fluorescein fluorescence intensity compared with adjacent tumor-infiltrated brain (Fig. 4). We also demonstrated a drop-off in fluorescein signal intensity at the tumor margins (Fig. 4).

To test the biodistribution of fluorescein without the confounding factor of the innate inflammatory response to xenografted cells, genetically induced tumors were established using the RCAS/tva system. Mouse pups harboring GFAP promoter-dependent expression of the virus receptor tva were successfully injected with cells producing RCAS virus containing an expression cassette for PDGFB

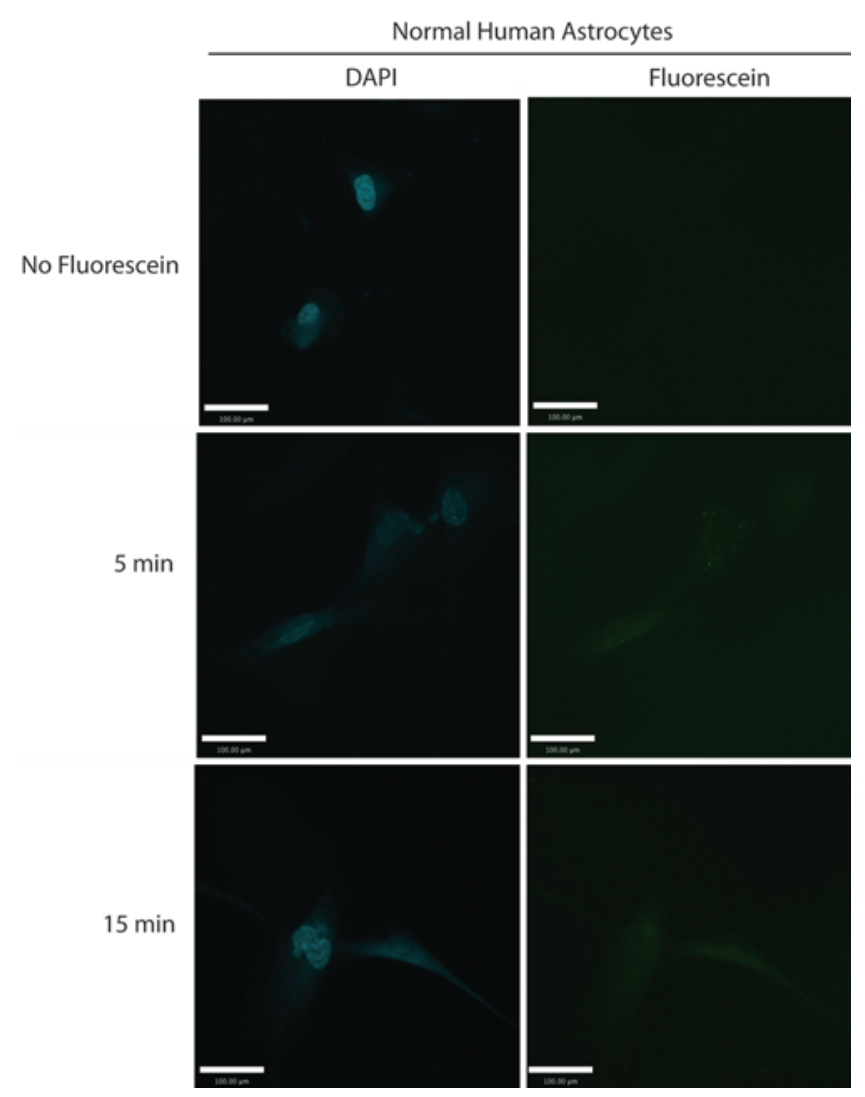

FIG. 1. Fluorescein uptake by NHAs after 5 and 15 minutes of exposure in vitro. Nuclear DNA stained with DAPI (blue) and fluorescein staining (green) of the cell body are observed. Bar $=100 \mu \mathrm{m}$. Figure is available in color online only.

and RFP. Cortical tumor formation was observed in 9 of 11 mice at 4-6 weeks. The tumor cells showed increased expression of GFAP and PDGFRA compared with glial cells and neurons in normal cortex (Fig. 5). Red fluorescence was observed on live cranial window imaging over the region of virus injection, and this signal was concordant with the presence of tumor immunolabeled for PDGFRA, GFAP, and RFP (Figs. 5 and 6). Dense glioma cell nests in this genetically induced glioma model demonstrated exclusion of fluorescein on cranial window imaging (Fig. 6). In regions in which cells were not densely clustered, fluorescein distribution to the adjacent brain parenchyma was observed, and this coincided with dextran extravasation (Fig. 6). We observed small-caliber vessels at the margins of tumor cell nests, which demonstrated early fluorescein and dextran extravasation, whereas in control animals that did not have tumor (PBS injected), fluorescein and dextran remained within cerebral vasculature. In Fig. 6, a strong fluorescein signal was observed at the margin of the imaging window in the control (PBS-injected) animal, and this coincided with dextran signal, which was attributed to tissue injury at the craniotomy margin. Fluorescein did not co-localize with glioma cells in either the xenograft or transgenic model. Thus, although fluorescein facilitates the demarcation of tumor infiltration to normal brain, it did not label glioma cells specifically. 

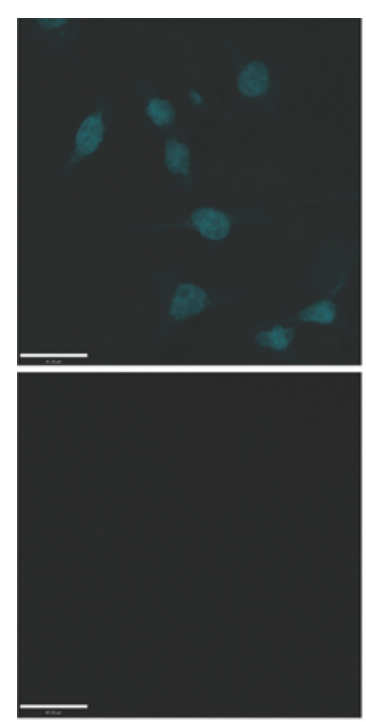

No Fluorescein

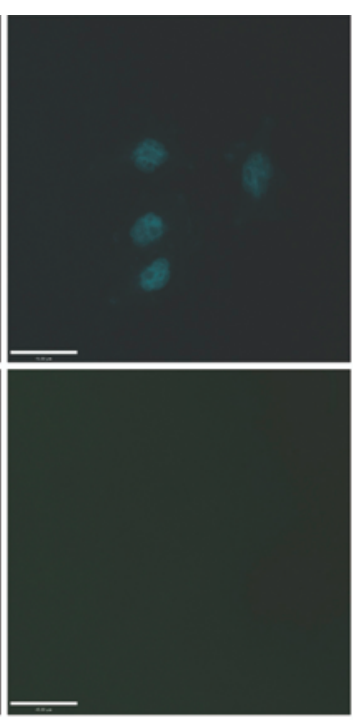

5 min

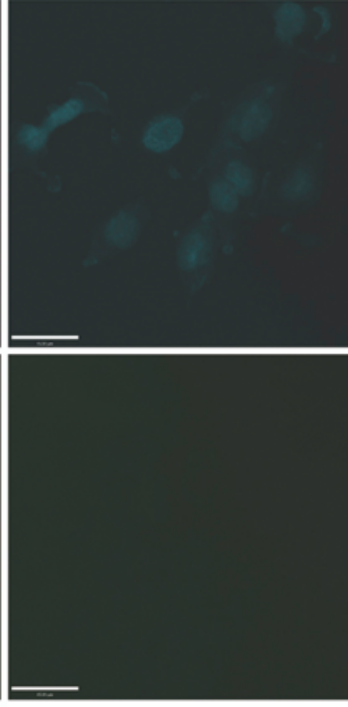

$15 \mathrm{~min}$
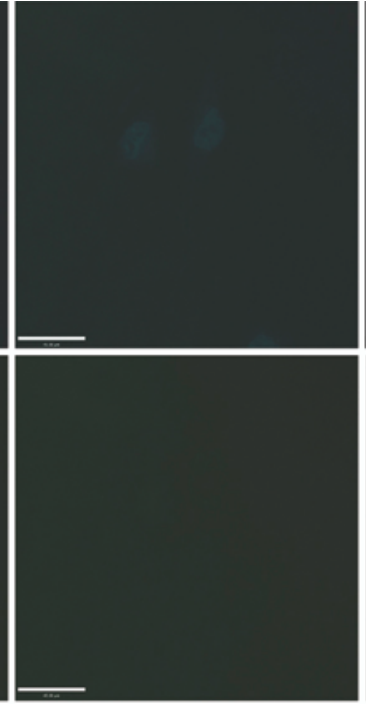

$30 \mathrm{~min}$

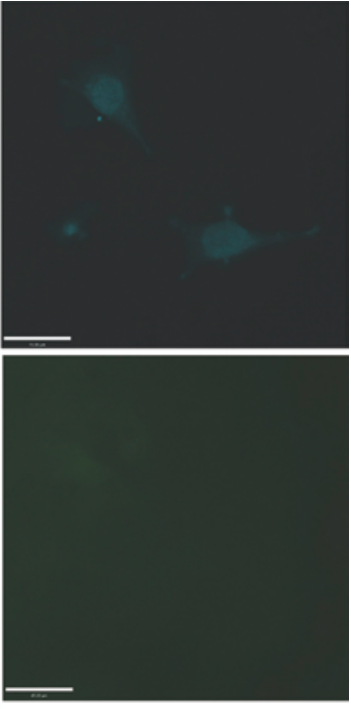

$60 \mathrm{~min}$

FIG. 2. Fluorescein distribution in U251 malignant glioma cell culture after $5,15,30$, and 60 minutes of exposure. Nuclear DNA stained with DAPI (blue). Fluorescein staining (green) is not enhanced in the nuclei or cell bodies of U251 cells compared with background. Some slight granular cytoplasmic staining is observed at 15 minutes but is absent at later time points. Bar $=45 \mu \mathrm{m}$. Figure is available in color online only.

\section{Clinical Study Methods}

Twelve patients with high-grade gliomas underwent fluorescein-guided resection of their tumors. Nine patients harbored a new tumor and 3 suffered from recurrent tumors. Patient characteristics are summarized in Table 2. Intravenous fluorescein is FDA approved for diagnostic angiography or angioscopy of the retina and iris vasculature. The protocol for the off-label use of fluorescein in this study was approved by the Indiana University Institutional Review Board. Informed consent was obtained from all subjects.

We administered a 3-mg/kg bolus of fluorescein sodium (AK-Fluor 10\%) intravenously through a peripheral venous line at the time of anesthesia induction. Once the dura was opened, the resection was performed using a Pentero 900 microscope with the microscope-integrated Yellow 560 camera (Meditec, Carl Zeiss). We have previously described the principles of fluorescein fluorescence and the technical details of the Yellow 560 module for resection of high-grade gliomas..$^{22}$ During our preliminary studies, we gradually decreased the dose of intravenous fluorescein in 10 patients while maintaining the fluorescent signal within the tumors. These preliminary results allowed us to minimize the required dose of fluorescein and potential side effects. The timing of fluorescein injection was also adjusted to allow reasonable clearance of the fluorescein from the intravascular space while avoiding fluorescein washout from the tumor by the time we started opening of the dura and resection of the tumors. This timing avoided leakage of fluorescein during resection caused by vascular wall injury and subsequent contamination of the field by the "false" fluorescent signal.

For each patient, most of the resection was performed under the Yellow 560 module, switching to white light only when significant bleeding was encountered. We also used neuronavigation (StealthStation, Medtronic Inc.) to guide resection and document the correlation between the fluorescence at the tumor margin and the margin of gadolinium enhancement on MRI. For each patient, multiple (3-8) random fluorescent and nonfluorescent samples were obtained at the tumor margins defined by the fluorescent and MR neuronavigation guidance. Areas that exhibited significant fluorescence at the gadolinium-enhancing margin based on neuronavigation data were biopsied and labeled "fluorescent," and then all fluorescent tissue was resected until very minimal fluorescence was appreciated. The nonfluorescent or minimally fluorescent margins after resection were also biopsied in multiple areas, and the corresponding specimens were labeled "nonfluorescent." No biopsies from the tumor core were obtained for the purposes of this study. The diagnosis of high-grade glioma was confirmed by frozen section intraoperatively. A postoperative gadolinium-enhanced MR image was obtained in each patient within 24 hours of resection. There were no intra- or postoperative complications related to the use of fluorescein. An independent neuropathologist blinded to the labeling of the specimens reviewed all samples, confirmed the diagnosis, and provided quantitative analysis of the percentage of viable tumor and necrosis for each specimen.

\section{Statistical Analysis}

A 2-tailed Fisher's exact test with a p value of $<0.05$ as significant was used to determine if fluorescence signal was associated with the presence of tumor on histology.

\section{Clinical Results}

Gross-total resection (GTR; Fig. 7) of the enhancing tumor was achieved in every case based on postoperative MRI examination. Histopathological analysis of the per- 

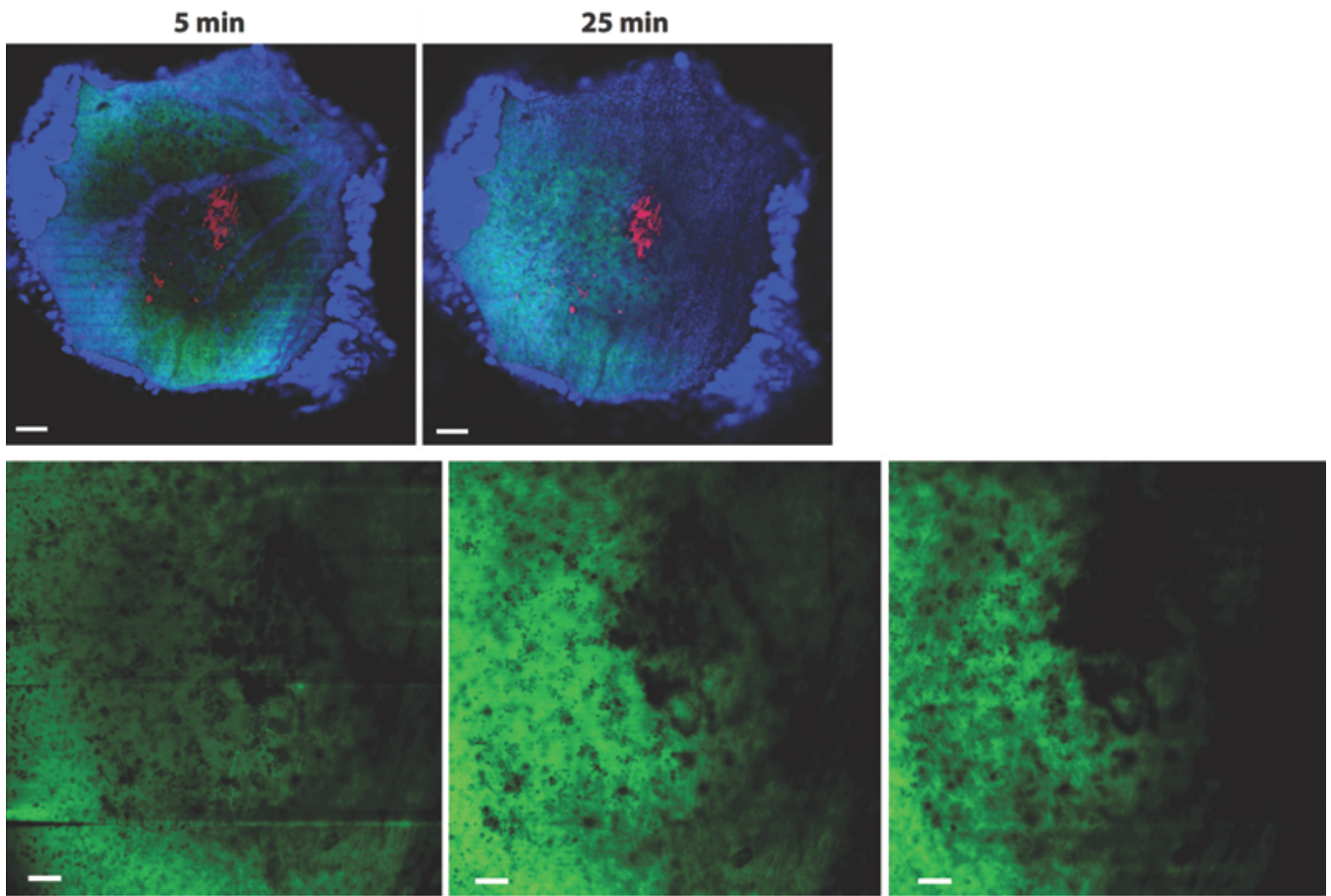

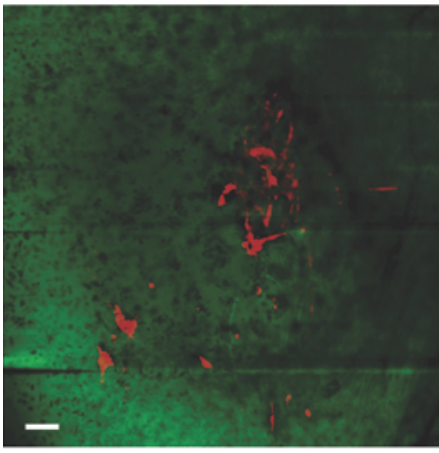

5 min

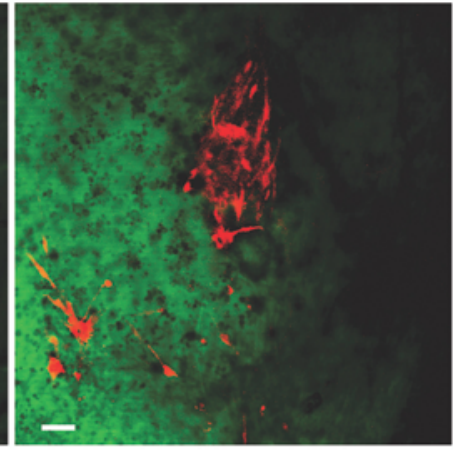

$15 \mathrm{~min}$

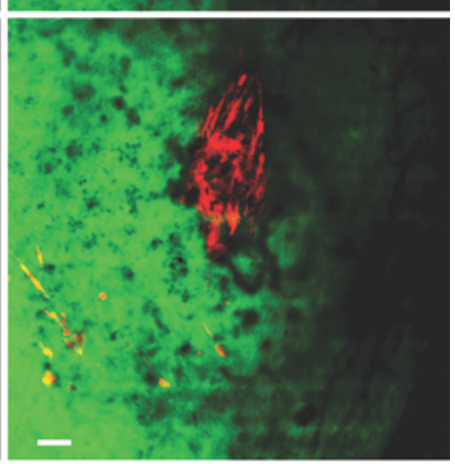

$25 \mathrm{~min}$

FIG. 3. Upper: In vivo cranial window fluorescence microscopy images at 5 minutes and 25 minutes after tail vein administration of fluorescein and Alexa Fluor 647 10-kD dextran. Red fluorescence represents mCherry (tumor cell) signal, blue fluorescence represents Alexa Fluor 647 dextran signal, and green fluorescence represents fluorescein signal. Note that the dextran extravasates into the brain parenchyma at 25 minutes, whereas fluorescein staining is seen at 5 minutes and becomes more diffuse by 25 minutes. Bar $=200 \mu \mathrm{m}$. Lower: Magnified in vivo cranial window images at 5, 15, and 25 minutes after fluorescein administration showing lack of fluorescein (green) uptake by engrafted U87 glioma cells (red). The upper row shows the fluorescein channel and the lower row shows the merge of mCherry and fluorescein channels. Bar $=100 \mu \mathrm{m}$. Figure is available in color online only.

manent sections was consistent with diagnosis of glioblastoma multiforme in 11 cases and gliosarcoma in 1 case. A total of 39 fluorescent and 28 nonfluorescent specimens were obtained. The median tumor content of the fluorescent specimens was $95 \%$ (range $0-100$; interquartile range [IQR] 50-100). The median proportion of necrotic tissue for the fluorescent specimens was 10\% (range 0-50; IQR 1-10). For the nonfluorescent specimens, the median content of identifiable glioma cells was $0 \%$ (range $0-100 ;$ IQR $0-13.75)$. The median necrotic content for nonfluorescent samples was $0 \%$ (range $0-5$; IQR 0 ). A false positive for a fluorescent specimen was defined as absence of tumor cells or nonspecific reactive changes within the fluorescent sample. Two of 39 samples were considered false positives. A false negative for a nonfluorescent sample was defined as presence of identifiable tumor within the speci- men. Eight of 28 specimens were considered false negatives. The presence of fluorescence in the tissue sample was significantly associated with presence of identifiable tumor $(p<0.0001)$. The details of the false positives and false negatives are summarized in Table 3 . The calculated sensitivity for fluorescein fluorescence to identify tumor based on these results was $82.2 \%$, whereas its specificity was $90.9 \%$.

\section{Discussion}

Moore $^{17,18}$ first reported the use of sodium fluorescein for demarcation of intracranial tumors in 1947. Subsequent experiments and efforts to determine the microscopic localization of fluorescein within intracranial tumor models were hindered by the highly diffusible and soluble nature 

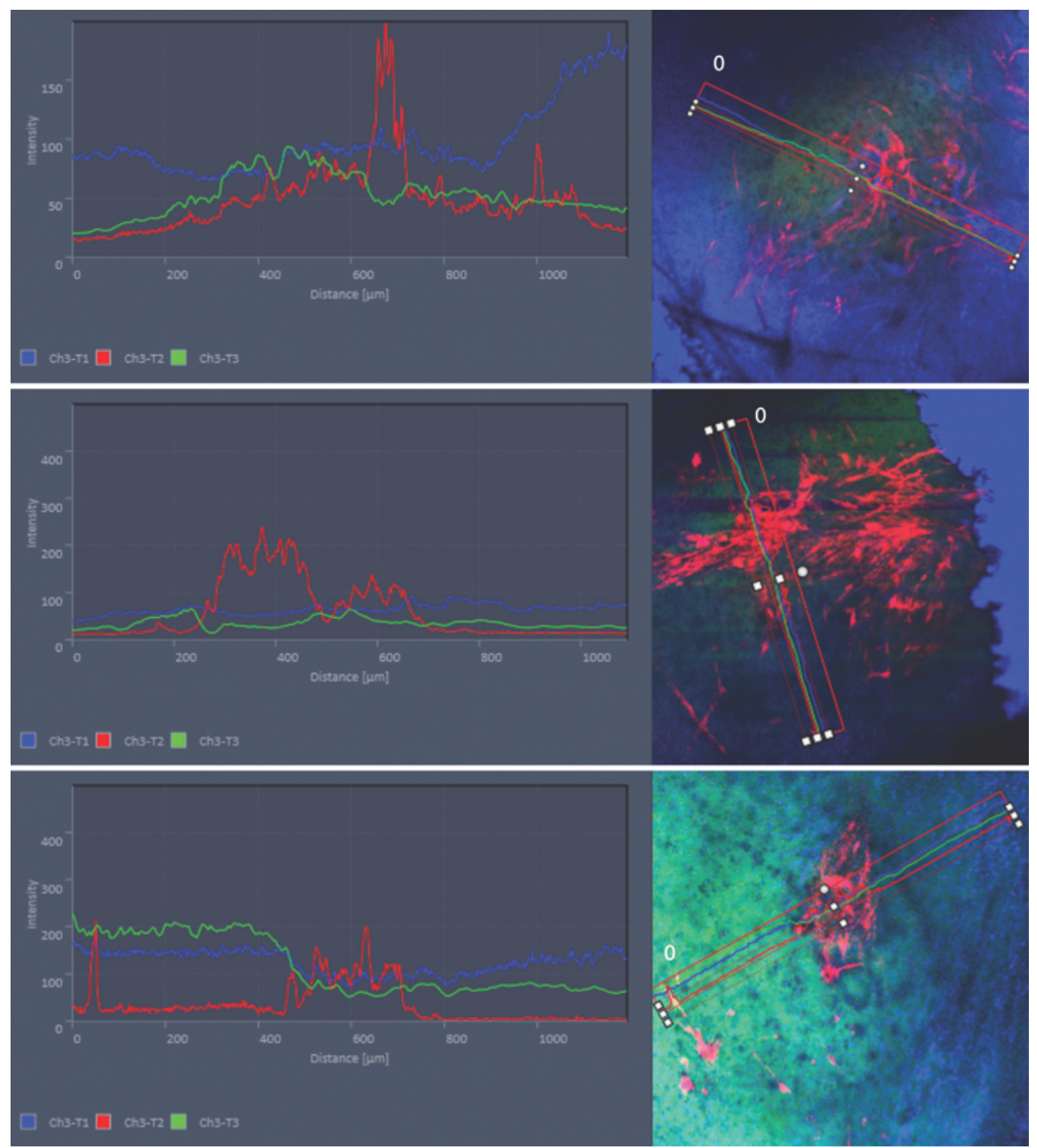

FIG. 4. In vivo fluorescence intensity measurements across tumor-infiltrated brain and adjacent parenchyma in 3 individual mice bearing U87 mCherry-expressing intracranial xenografts. The images were obtained 15 minutes after fluorescein administration at $10 \times$ magnification. Fluorescence intensity graphs are on the left and represent the intensity at intervals along the linear region of interest demonstrated on the merged 3-channel image on the right. Red fluorescence represents mCherry (tumor cell) signal, blue fluorescence represents Alexa Fluor 647 dextran signal, and green fluorescence represents fluorescein signal. Figure is available in color online only.

of fluorescein. In fact, only the distribution of fluorescein conjugated to albumin in fixed tissue sections and not fluorescein alone was reported..$^{13}$ Our experimental results suggest that fluorescein demarcation of glioma-invaded brain is the result of distribution of fluorescein into the extracellular spaces, likely as a result of abnormal BBB permeability in the tumor-affected brain. We did not observe a glioblastoma tumor cell-specific uptake of fluorescein. In fact, tumor cells appear to mostly exclude fluorescein. Our results are consistent with the observation of Ichioka et al. ${ }^{9}$ who reported an absence of increased fluorescence signal in U251 glioblastoma flank xenografts compared with normal tissue after intravenous administration of fluorescein. Furthermore, our results confirm the observation of fluorescein staining in the region of tumor in a C6 rat glioma model ${ }^{10}$ Marked fluorescence in tissue that enhances on
CT or MRI has been shown previously in patients who harbor malignant glioma. ${ }^{15}$ The region of marked fluorescence was associated histologically with dense cellular clusters, whereas regions with sparse cells did not demonstrate fluorescence..$^{15}$ Fluorescein signal in the brain of pigs and rodents is minimal unless BBB permeability is enhanced. ${ }^{1,2,14}$ Furthermore, rodent BBB disruption with intraarterial mannitol administration has been demonstrated to enhance fluorescein signal in the brain. ${ }^{8}$ Given these observations, we can explain the tumor field demarcation effect created by fluorescein as a product of altered BBB permeability and extracellular leakage of fluorescein within the infiltrative tumor. As such, resection based on fluorescein fluorescence would only accomplish the goal of removing the volume of brain with disrupted BBB dynamics and not necessarily the full extent of infiltrating tumor 




FIG. 5. Left: Low-magnification coronal sections at the site of cranial window imaging in Fig. 6 reveal a glioma in the virus-injected right hemisphere that immunolabels for GFAP, PDGFRA, and RFP. Bar $=2$ $\mathrm{mm}$. Sections are oriented so the top is dorsal and the bottom is ventral. Right: Magnified (20x) image of tumor cells in the right hemisphere stained with H\&E or immunolabeled for GFAP, PDGFR, or RFP. Bar = $100 \mu \mathrm{m}$. Figure is available in color online only.

cells. The necrotic portion of the tumor does not stain with fluorescein due to its lack of cerebral vasculature.

The use of fluorescein fluorescence to demarcate malignant gliomas should not be viewed as a property specific to glioblastoma tumor cells and thus cannot be defined as a specific marker for resection of glioblastoma. This fluorescence technology is a marker of BBB breakdown corresponding to the area of contrast enhancement on the MRI. Given the practical applicability of this form of microscope-integrated camera and the presence of fluorescein within the region of tumor invasion associated with increased vascular permeability, this technology may provide surgeons with another adjunct for surgical planning and resection of malignant gliomas. Gross-total resection of glioma has been reported in 8 of 10 patients with the use of fluorescein administered at a dose of $15-20 \mathrm{mg} / \mathrm{kg}$ intraoperatively compared with 4 of 12 patients without the use of fluorescein. ${ }^{5}$ These authors demonstrated progression-free survival of 7.2 months in the group who underwent fluorescence-guided resection versus 5.4 months in the group in whom conventional white-light resection was employed. However, the presence of WHO Grade II, III, and IV tumors in each of the study groups confounds the interpretation of the effect of fluorescein use in improving progression-free survival.

In a prospective series of glioblastomas resected with or without fluorescein fluorescence as an adjunct, the rate of GTR increased by $28 \%$ and the median survival time slightly increased by 2 weeks with no statistically significant difference in overall survival. ${ }^{11}$ In our series, GTR was achieved in every patient. The use of a specific microscope-integrated camera for detection of fluorescein fluorescence may allow for a more accurate demarcation of tumor margins than tumor margins observed under white light or with the application of high doses of fluorescein without the use of an operating microscope, as reported in previous studies. ${ }^{5,11,19,24}$ The use of lower doses of fluorescein also decreases the potential complications associated with higher doses. ${ }^{7}$ Based on our analysis, the histopathological analysis of the fluorescent intraoperative samples correlated well with the laboratory in vivo findings. Our results reveal that fluorescein fluorescence is highly specific (90.9\% in our series) with very low false positives. As demonstrated in the laboratory experiment, fluorescein accumulates in areas of the brain with disrupted BBB. These areas are likely to contain malignant cells. The calculated sensitivity is, however, lower ( $82.2 \%$ in our study). This is likely because the infiltrating edge of the high-grade glioma might not have disrupted the BBB effectively; therefore, fluorescein will not accumulate in these areas even though malignant cells are present.

In our animal model, we found that, due to fluorescein exclusion by tumor cells, areas infiltrated with dense clusters of cells might not exhibit fluorescence. This phenomenon might explain some of the false negatives found to have high focal infiltration (see Table 3 for details). Based on our intraoperative observations, neuronavigation confirmed in every case that the fluorescent edge of the tumor corresponded to the edge of the gadolinium-enhancing area on the preoperative MR image. We sampled the margins of the tumor where the fluorescent signal can be least reliable. Nonetheless, fluorescein fluorescence has demonstrated its potential candidacy as a useful adjunct in resection of the enhancing portion of high-grade gliomas. Larger-scale studies are necessary to determine the efficacy of this technology in improving extent of resection as well as progression-free and overall patient survival.

\section{Conclusions}

The absence of robust bioaccumulation of sodium fluorescein in vitro and in vivo within malignant glioma cells indicates that fluorescein staining of glioma-infiltrated brain regions is a consequence of non-cell-specific mechanisms. We demonstrated rapid distribution of fluorescein into the brain parenchyma infiltrated by human glioblastoma xenograft cells or PDGFB-induced glioma in mouse followed by extravasation of $10-\mathrm{kD}$ dextran. This suggests that local factors such as BBB disruption may contribute to the tumor-region demarcation phenomenon observed intraoperatively. We refute the hypothesis from which we started that enhanced uptake and slow washout of fluorescein is due to selective uptake of fluorescein into glioma cells. 


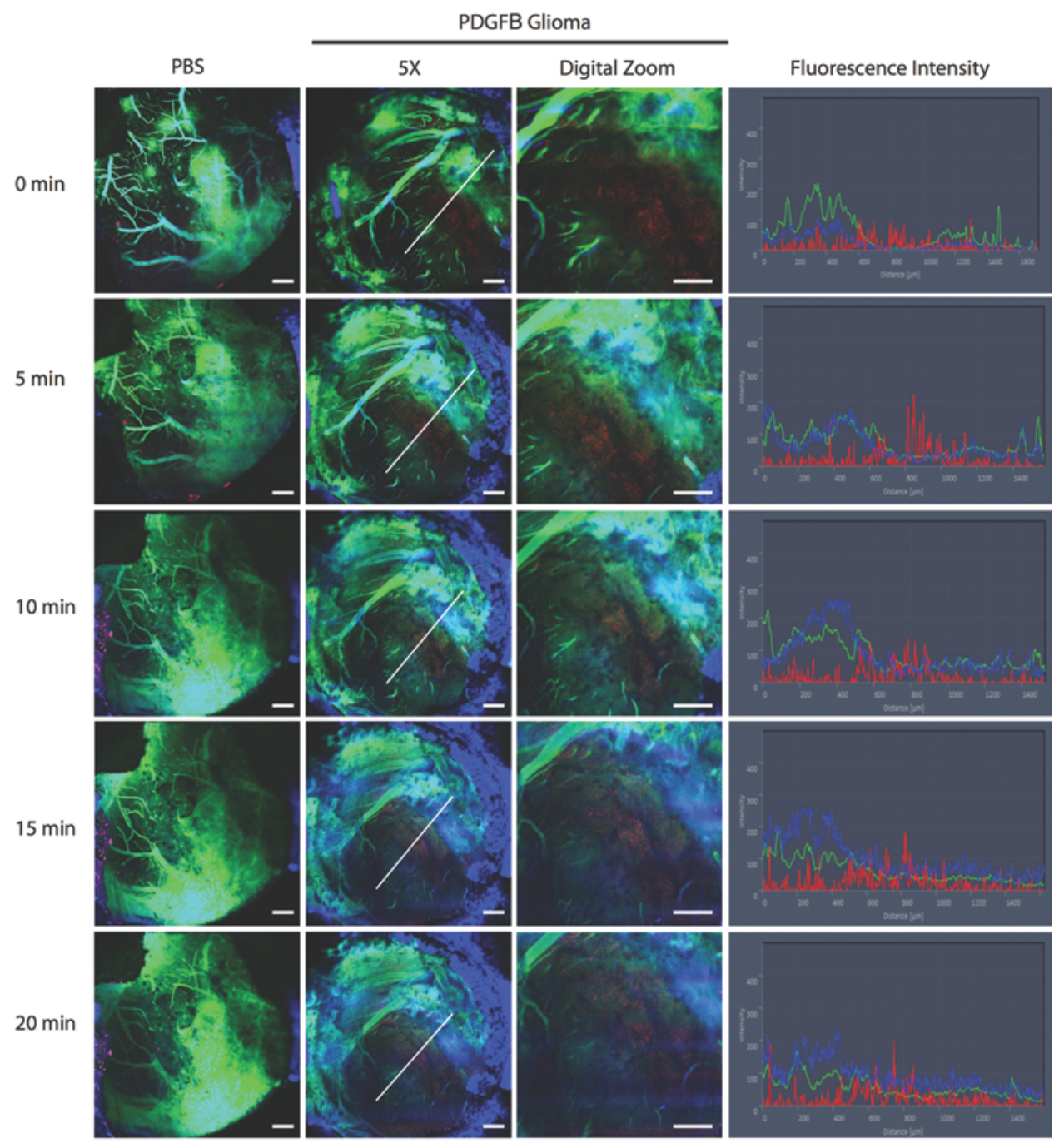

FIG. 6. In vivo cranial window fluorescence microscopy of fluorescein biodistribution into brain after intravenous administration. The right frontal lobe of mice is visualized 4 weeks after injection of PBS or RCAS-PDGFB-RFP viral injection. Transformed cells (glioma) express RFP and are labeled in red; fluorescein is labeled in green; and Alexa Fluor 647 10-kD dextran is labeled in blue. Fluorescence intensity measurements for RFP (red), fluorescein (green), and Alexa Fluor 647 dextran (blue) fluorescence are graphed against the distance along the linear region of interest with origin on the left of the white line shown. Bar $=200 \mu \mathrm{m}$. Figure is available in color online only.

\section{TABLE 2. Patient characteristics and histology}

\begin{tabular}{rrlll}
\hline Case No. & Age (yrs), Sex & \multicolumn{1}{c}{ Tumor Location } & Pathology Diagnosis & Resection \\
\hline 1 & $86, \mathrm{M}$ & Lt anterior temporal & Glioblastoma, new Dx & GTR \\
\hline 2 & $58, \mathrm{M}$ & Rt posterior temporal & Glioblastoma, recurrent & GTR \\
\hline 3 & $67, \mathrm{M}$ & Rt temporal-frontal operculum & Glioblastoma, new Dx & GTR \\
\hline 4 & $45, \mathrm{M}$ & Lt posterior temporal & Glioblastoma, recurrent & GTR \\
\hline 5 & $54, \mathrm{M}$ & Lt occipital & Glioblastoma, new Dx & GTR \\
\hline 6 & $47, \mathrm{M}$ & Lt temporal operculum & Glioblastoma, recurrent & GTR \\
\hline 7 & $51, \mathrm{M}$ & Lt temporal & Glioblastoma, new Dx & GTR \\
\hline 8 & $59, \mathrm{M}$ & Rt temporooccipital & Gliosarcoma, new Dx & GTR \\
\hline 9 & $82, \mathrm{M}$ & Rt temporal & Glioblastoma, new Dx & GTR \\
\hline 10 & $67, \mathrm{M}$ & Rt occipital & Glioblastoma, new Dx & GTR \\
\hline 11 & $56, \mathrm{~F}$ & Lt parietooccipital & Glioblastoma, new Dx & GTR \\
\hline 12 & $51, \mathrm{~F}$ & Rt parietal & Glioblastoma, new Dx & GTR \\
\hline
\end{tabular}

$\mathrm{Dx}=$ diagnosis; $\mathrm{GTR}=$ gross-total resection. 


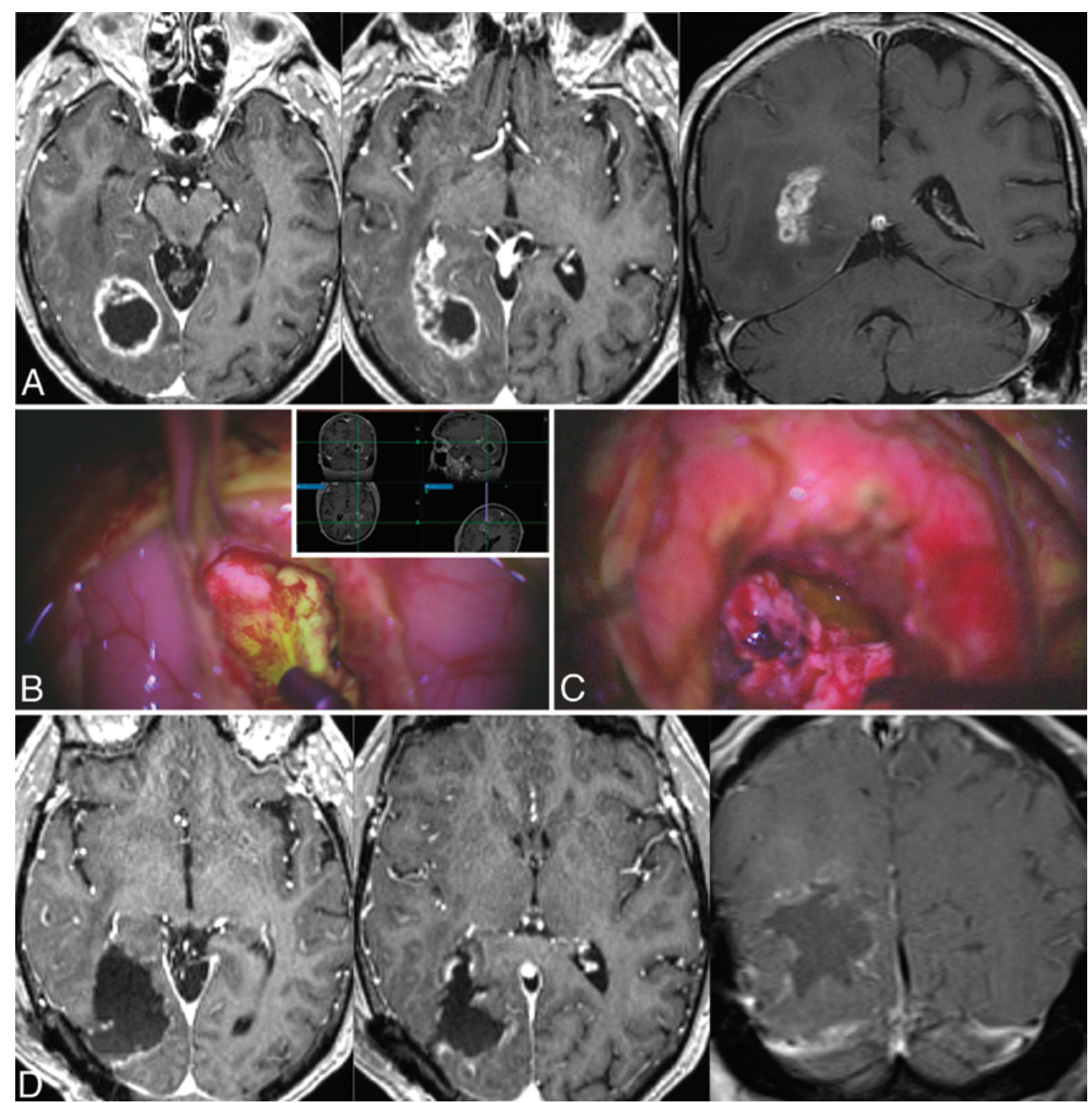

FIG. 7. A: A right occipital glioblastoma in a 67-year-old man presenting with visual decline. B: The fluorescent margin of the tumor against the normal brain is demonstrated; note the correlation between the fluorescent margin and the enhancing margin of the tumor based on intraoperative neuronavigation data (inset). C: Also note minimal fluorescent signal in the resection cavity and CSF, filling the defect. D: Postoperative MRI revealing gross-total removal of the enhancing region extending into the ventricle. Figure is available in color online only.

\section{TABLE 3. Fluorescence and histology correlation}

\begin{tabular}{ccccl}
\hline Case No. & Fluorescent Specimens & False Positive & Nonfluorescent Specimens & False Negative \\
\hline 1 & 2 & 0 & 3 & 0 \\
\hline 2 & 10 & 2 (gliosis) & 6 & 1 (10\% focal glioblastoma) \\
\hline 3 & 2 & 0 & 2 & 0 \\
\hline 4 & 5 & 0 & 2 & 1 (20\% glioblastoma, $5 \%$ necrosis) \\
\hline 6 & 1 & 0 & 1 & 1 (100\% glioblastoma) \\
\hline 7 & 2 & 0 & 1 & 1 (Infl edge 20\%) \\
\hline 8 & 1 & 0 & 1 & 1 (50\% tumor infiltration) \\
\hline 9 & 5 & 0 & 4 & 0 \\
\hline 10 & 4 & 0 & 2 & 0 \\
\hline 11 & 2 & 0 & 2 & 1 (Infl edge 10\%) \\
\hline 12 & 2 & 0 & 2 & 0 \\
\hline Total & 3 & 0 & 2 & 8 \\
\hline
\end{tabular}

Infl edge = infiltrating edge of glioblastoma. 
Our intraoperative observations and histopathological analysis demonstrate a good correlation between intraoperative fluorescein fluorescence and gadolinium enhancement on MR imaging. Even though fluorescein sodium does not appear to be a tumor-specific fluorophore, intraoperative fluorescein fluorescence is a simple and practical tool that can help the neurosurgeon increase the rates of GTR for high-grade gliomas.

\section{References}

1. Ábrahám CS, Deli MA, Joó F, Megyeri P, Torpier G: Intracarotid tumor necrosis factor-alpha administration increases the blood-brain barrier permeability in cerebral cortex of the newborn pig: quantitative aspects of double-labelling studies and confocal laser scanning analysis. Neurosci Lett 208:8588, 1996

2. Abrahám CS, Harada N, Deli MA, Niwa M: Transient forebrain ischemia increases the blood-brain barrier permeability for albumin in stroke-prone spontaneously hypertensive rats. Cell Mol Neurobiol 22:455-462, 2002

3. Becher OJ, Hambardzumyan D, Walker TR, Helmy K, Nazarian J, Albrecht S, et al: Preclinical evaluation of radiation and perifosine in a genetically and histologically accurate model of brainstem glioma. Cancer Res 70:2548-2557, 2010

4. Burrell K, Hill RP, Zadeh G: High-resolution in-vivo analysis of normal brain response to cranial irradiation. PLoS ONE 7:e38366, 2012

5. Chen B, Wang H, Ge P, Zhao J, Li W, Gu H, et al: Gross total resection of glioma with the intraoperative fluorescenceguidance of fluorescein sodium. Int J Med Sci 9:708-714, 2012

6. da Silva CE, da Silva JL, da Silva VD: Use of sodium fluorescein in skull base tumors. Surg Neurol Int 1:70, 2010

7. Dilek O, Ihsan A, Tulay H: Anaphylactic reaction after fluorescein sodium administration during intracranial surgery. $\mathbf{J}$ Clin Neurosci 18:430-431, 2011

8. Hawkins BT, Egleton RD: Fluorescence imaging of bloodbrain barrier disruption. J Neurosci Methods 151:262-267, 2006

9. Ichioka T, Miyatake S, Asai N, Kajimoto Y, Nakagawa T, Hayashi $\mathrm{H}$, et al: Enhanced detection of malignant glioma xenograft by fluorescein-human serum albumin conjugate. $\mathbf{J}$ Neurooncol 67:47-52, 2004

10. Kabuto M, Kubota T, Kobayashi H, Nakagawa T, Ishii H, Takeuchi H, et al: Experimental and clinical study of detection of glioma at surgery using fluorescent imaging by a surgical microscope after fluorescein administration. Neurol Res 19:9-16, 1997

11. Koc K, Anik I, Cabuk B, Ceylan S: Fluorescein sodiumguided surgery in glioblastoma multiforme: a prospective evaluation. Br J Neurosurg 22:99-103, 2008

12. Kremer P, Wunder A, Sinn H, Haase T, Rheinwald M, Zillmann U, et al: Laser-induced fluorescence detection of malignant gliomas using fluorescein-labeled serum albumin: experimental and preliminary clinical results. Neurol Res 22:481-489, 2000

13. Krigman MR, Manuelidis EE: Distribution of fluorescent tracers in heterologously transplanted intracerebral tumors. Cancer Res 24:1749-1759, 1964

14. Kunes M, Kvetina J, Malakova J, Bures J, Kopacova M, Rejchrt S: Pharmacokinetics and organ distribution of fluorescein in experimental pigs: an input study for confocal laser endomicroscopy of the gastrointestinal tract. Neuroendocrinol Lett 31 (Suppl 2):57-61, 2010

15. Kuroiwa T, Kajimoto Y, Ohta T: Comparison between operative findings on malignant glioma by a fluorescein surgical microscopy and histological findings. Neurol Res 21:130134, 1999

16. Kuroiwa T, Kajimoto Y, Ohta T: Development of a fluorescein operative microscope for use during malignant glioma surgery: a technical note and preliminary report. Surg Neurol 50:41-49, 1998

17. Moore GE: Fluorescein as an agent in the differentiation of normal and malignant tissues. Science 106:130-131, 1947

18. Moore GE, Peyton WT, French LA, Walker WW: The clinical use of fluorescein in neurosurgery; the localization of brain tumors. J Neurosurg 5:392-398, 1948

19. Murray KJ: Improved surgical resection of human brain tumors: Part I. A preliminary study. Surg Neurol 17:316-319, 1982

20. O'goshi K, Serup J: Safety of sodium fluorescein for in vivo study of skin. Skin Res Technol 12:155-161, 2006

21. Okuda T, Kataoka K, Yabuuchi T, Yugami H, Kato A: Fluorescence-guided surgery of metastatic brain tumors using fluorescein sodium. J Clin Neurosci 17:118-121, 2010

22. Rey-Dios R, Cohen-Gadol AA: Technical principles and neurosurgical applications of fluorescein fluorescence using a microscope-integrated fluorescence module. Acta Neurochir (Wien) 155:701-706, 2013

23. Schebesch KM, Proescholdt M, Höhne J, Hohenberger C, Hansen E, Riemenschneider MJ, et al: Sodium fluoresceinguided resection under the YELLOW $560 \mathrm{~nm}$ surgical microscope filter in malignant brain tumor surgery-a feasibility study. Acta Neurochir (Wien) 155:693-699, 2013

24. Shinoda J, Yano H, Yoshimura S, Okumura A, Kaku Y, Iwama T, et al: Fluorescence-guided resection of glioblastoma multiforme by using high-dose fluorescein sodium. Technical note. J Neurosurg 99:597-603, 2003

25. Sjöback R, Nygren J, Kubista M: Absorption and fluorescence properties of fluorescein. Spectrochim Acta A Mol Biomol Spectrose 51:L7-L21, 1995

\section{Author Contributions}

Conception and design: all authors. Acquisition of data: all authors. Analysis and interpretation of data: all authors. Drafting the article: all authors. Critically revising the article: all authors. Reviewed submitted version of manuscript: all authors. Approved the final version of the manuscript on behalf of all authors: Cohen-Gadol.

\section{Correspondence}

Aaron A. Cohen-Gadol, Goodman Campbell Brain and Spine, Department of Neurological Surgery, Indiana University, $355 \mathrm{~W}$. 16th St., Ste. 5100, Indianapolis, IN 46202. email: acohenmd@ gmail.com. 